Maria Roussos
Andrew Johnson
Thomas Moher
Jason Leigh
Christina Vasilakis
Craig Barnes
nice@ice.eecs.uic.edu
www.ice.eecs.uic.edu/ nice
Electronic Visualization Laboratory
(EVL) and
Interactive Computing Environments
Laboratory (ICE)
University of Illinois at Chicago
851 S. Morgan St., Room 1120
Chicago, IL 60607

\section{Learning and Building Together in an Immersive Virtual World}

\begin{abstract}
A bstract
This paper describes the design, evaluation, and lessons learned from a project involving the implementation of an immersive virtual environment for children called N ICE ( $N$ arrative-based, Immersive, Constructionist/Collaborative Environments). The goal of the N ICE project was to construct a testbed for the exploration of virtual reality as a learning medium within the context of the primary educational reform themes of the past three decades. W ith a focus on informal education and domains with social content, N ICE embraces the constructivist approach to learning, collaboration, and narrative develo pment, and is designed to utilize the strengths of virtual reality: a combination of immersion, telepresence, immediate visual feedback, and interactivity. Based on our experiences with a broad range of users, the paper discusses both the successes and limitations of N ICE and concludes with recommendations for research directions in the application of immersive VR technologies to children's learning.
\end{abstract}

\section{Introduction}

There are good reasons to presume that the application of virtual reality (VR) technologies to children's conceptual learning is, in the words of Fred Brooks, "rank foolishness"' (Brooks, 1998). To date, there exists no clear evidence that VR brings "added value" to learning in children, and historical experience with other media offers scant hope for powerful effects (Clark, 1983; Cuban, 1986). Even if overwhelming evidence of effectiveness were available, the prohibitive costs of VR technologies and concomitant staff development, operations, and maintenance would find no place in dwindling school budgets that are overwhelmingly dominated by human resource costs. Price/ performance issues aside, there remain strong objections among educators and developmental psychologists regarding the appropriateness of virtual experiences for children (Cuban, 1986).

Yet, in spite of these concerns, there remain compelling reasons for believing that VR learning environments for children warrant serious investigation. There is general agreement that VR environments can have strong motivational impact (Bricken, 1991), and ongoing efforts at characterizing phenomena such as immersion and presence are beginning to clarify these effects (Winn, 1993; Slater \& Wilbur, 1997). VR affords opportunities to experience environments that, for reasons of time, distance, scale, and safety, would not otherwise be available to many young children, especially those with disabilities (Cromby, Standen, \& Brown, 1995). Early exposure to virtual environments may both leverage the well-known efficiency and capacity of children's learning and provide advance organizers for later learning experiences ( $D$ ede, 1998). U sability 
issues that plague adult VR users may prove less problematic among children, who both easily adapt to graphic and conceptual abstraction (in cartoons and comics) and who often have extensive experience in navigating 3-D spaces and discovering and exercising interface affordances (Provenzo, 1991).

In this paper, we describe our experience in the development and assessment of the distributed virtual reality environment NICE ( $\mathrm{N}$ arrative, I mmersive, Collaborative/ Constructivist Environment), designed to support children's learning of simple relationships between plant growth, sunlight, and water. N ICE implements a persistent virtual garden in which children may collaboratively plant and harvest fruits and vegetables, cull weeds, and position light and water sources to differentially affect the growth rate of plants. NICE has been operational since July of 1996, and has now been "visited" by well over 300 users from around the world.

We begin with a brief survey of the use of VR technologies in support of learning. $N$ ext, we describe the N ICE world, the learning and pedagogical themes that informed its design, and briefly discuss its implementation. A major portion of the paper is devoted to user experience and formal assessment of NICE as a learning environment. Finally, we close with a discussion of lessons learned from the NICE project, and how our experience with NICE is shaping our future research directions.

\section{Children's Learning in Immersive Virtual Environments}

Research in conceptual learning and virtual reality is a relatively young field, but it is growing rapidly. In a recent report by the Institute for D efense Analysis, Christine Youngblut comprehensively surveys work over the past few years in the area, citing approximately fifty VR-based learning applications that include desktop but exclude text-based virtual environments (Youngblut, 1998). We restrict our focus here to those projects involving immersive VR technologies that are applied specifically to elementary and middle-school children's learning.
The H uman I nterface Technology L aboratory ( $H I T L)$ at the $U$ niversity of Washington has been one of the early educational seed beds for VR, with projects such as the Virtual Reality Roving Vehicle (VRRV) (R ose, 1995; Winn, 1993) and summer camp programs in VR for students (Bricken \& Byrne, 1993). The VRRV project was experienced by a large number of students, while the summer camps focused on "world-building" activity, in which students conceived and created the objects of their own virtual worlds, using 3-D modeling software on desktop computers. Although this gave the opportunity for students to understand the process involved in creating a virtual setting, the actual immersive experience was limited to a short visit of the predesigned virtual worlds (ten-minute VR experiences), making it difficult to come to conclusions on the value of a virtual experience itself for conceptual learning.

The $\mathrm{N}$ ewtonWorld and $\mathrm{M}$ axwellWorld ScienceSpace projects developed by researchers at G eorge M ason U niversity and the $U$ niversity of H ouston (D ede, Salzman, \& L oftin, 1996) provide immersive learning environments in which students may explore the kinematics and dynamics of motion, electrostatic forces, and other physics concepts. Formative evaluation studies of these virtual worlds have been conducted with respect to their usability and learnability. These studies report on learners' engagement, surprise, and understanding of the alternative representations of the concepts provided in the ScienceSpace worlds (D ede et al., 1996). L imitations and discomfort caused by the current VR head-mounted displays hindered usability and learning. $\mathrm{O} n$ the other hand, multisensory cues, multimodal interaction, and the introduction of multiple new representations are believed to have helped students develop correct mental models of the abstract material.

Researchers at the Computer M useum developed an immersive VR application designed to teach children about the structure and function of cells ( $G$ ay $\&$ Greschler, 1994). In the application, users were asked to construct cells from component parts, with successful completion indicated by an animation of internal cell function. In a comparison between immersive and nonimmersive treatment groups, immersive subjects (children and adults) demonstrated better retention of sym- 
bolic information (remembering the names and functions of the organelles) and indicated more interest in taking a free biology class as a result of the experience.

Another exhibit-based research project, the Virtual Gorilla project (Allison, Wills, Bowman, Wineman, \& $\mathrm{H}$ odges, 1997) re-creates the gorilla exhibit at Zoo Atlanta, allowing users to adopt the role of an adolescent gorilla, navigating the environment and observing other gorillas' reactions to their approach. While no formal assessment has been reported, interviews with users elicited favorable responses in the sense of immersion, enjoyment, and successful communication of learning goals.

The above virtual worlds have been implemented to support only one (physically present or remote) student at a time. To our knowledge, the N ICE project is the first immersive, multiuser learning environment designed specifically for children.

\section{The NICE project}

The $\mathrm{N}$ arrative I mmersive Constructionist/ Collaborative Environments (NICE) project is an exploratory learning environment for children between the ages of six and ten (Roussos, J ohnson, L eigh, Barnes, Vasilakis, \& M oher, 1997, 1997b; J ohnson, Roussos, Leigh, Vasilakis, Barnes, \& M oher, 1998). The children's main activity in NICE is to collaboratively construct, cultivate, and tend a healthy virtual garden (Figure 1.) This activity takes place in a highly graphical immersive VR system called the CAVE (C ruz-N eira, Sandin, \& D eFanti, 1993).

The CAVE is a multiperson, room-sized VR system consisting of three walls and a floor. All users entering the CAVE wear special lightweight stereoglasses, which allow them to see both the virtual and the physical world unobtrusively, and use a lightweight hand-held wand for interaction (Figure 2). As the CAVE supports multiple simultaneous physical users, five to six children can participate in the learning activities at the same time. A similar but smaller VR system, the I mmersaD esk ${ }^{\mathbb{N i}}$, consists of one back-projected panel tilted at a $45 \mathrm{deg}$. angle and resembles a drafting table.

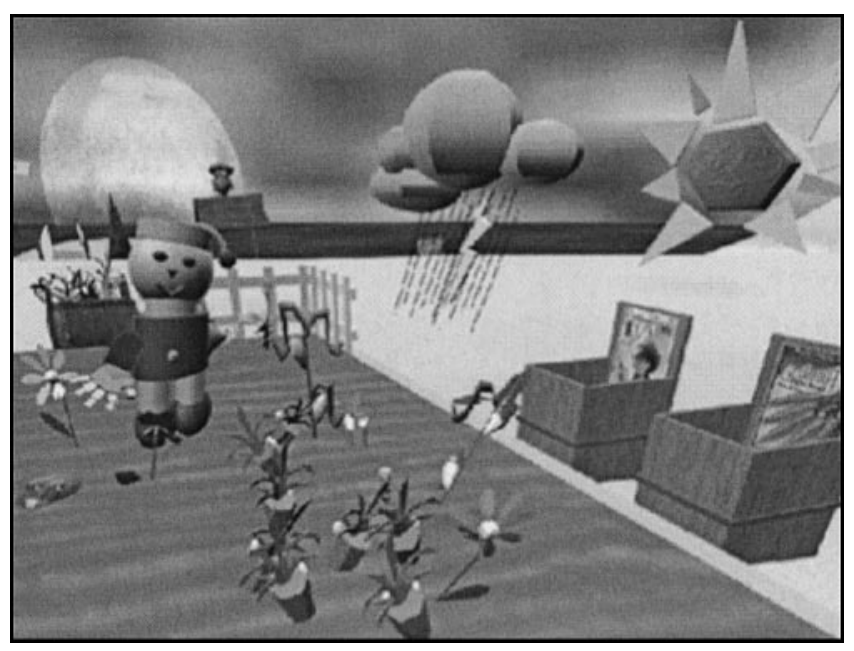

Figure 1. A child (represented by an avatar) planting in the N ICE garden.

The N ICE garden was originally designed as an environment for young children to learn about the effects of sunlight and rainfall on plants, the growth of weeds, the ability to recycle dead vegetation, and similar simple biological concepts that are a part of the life cycle of a garden. Since these concepts can be experienced by most children in a real garden, the NICE garden provides its users with tools that allow its exploration from multiple different perspectives. In addition to planting, growing, and picking vegetables and flowers, the children have the ability to shrink down and walk beneath the surface of the soil to observe the roots of their plants or to meet other underground dwellers. They can also leap high up in the air, climb over objects, factor time, and experience firsthand the effects of sunlight and rainfall by controlling the environmental variables that cause them.

Familiar methods of interaction are employed, that eliminating the use of menus by instead using simple visual metaphors. The wand has a joystick for navigation and three buttons: one for picking and planting, one for changing size, and one for leaping. In the garden, the children can choose from several crates of seeds. U sing the wand, a child can pick a seed from a crate and drop it onto the soil. The corresponding vegetable will then begin to grow. The children must make sure the plants are not too close together, and that they get enough water and sunlight. $U$ sing the same pick-and-place ac- 

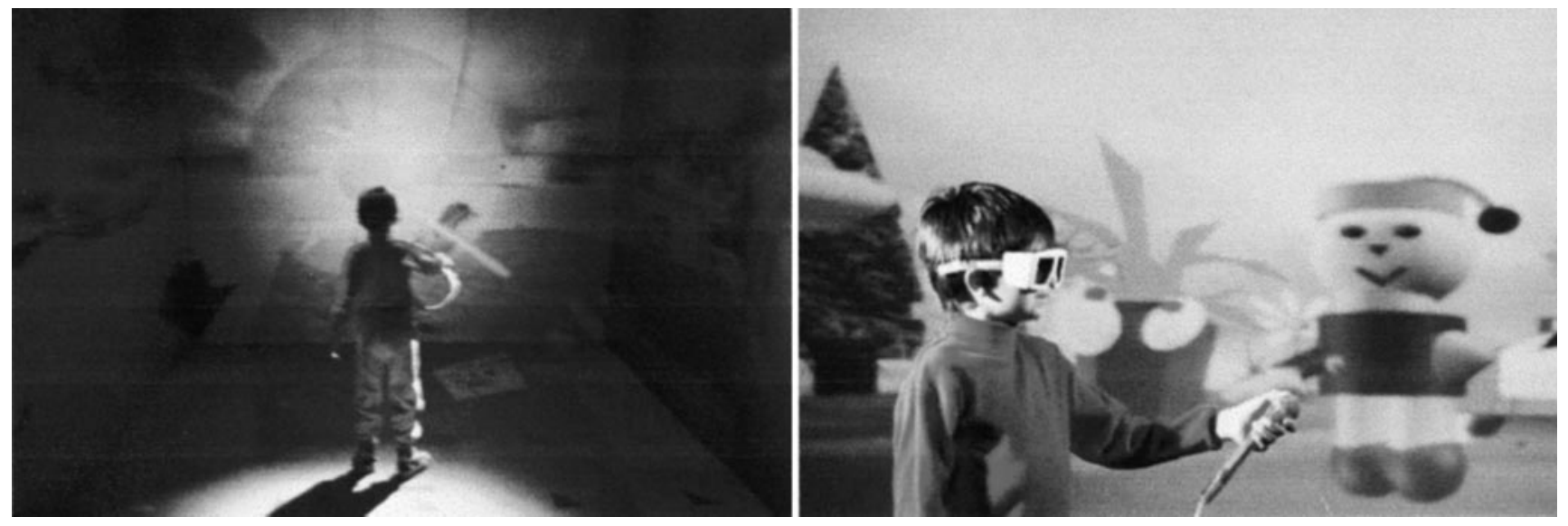

Figure 2. Eddie interacting with the NICE garden in the CAVE.

tion, they can water their plants by pulling a raincloud over them, provide sunlight with the use of the sun, or clear the garden weeds by recycling them in the compost heap. The symbolic representations of the various environmental elements as well as instant feedback are used to facilitate the learner's understanding of the biological relationships that take place in the garden. Thus, when the raincloud has been over a plant for too long, the plant holds an umbrella; when it's too sunny, it wears sunglasses, and so on.

The garden is persistent in that it continues to evolve, so the participants can return and check on its progress at a later time. The current garden has been growing for two years. In addition to the garden, the children have a whole island to explore: they can climb down a dormant volcano to access the catacombs beneath the island, look for fish in the sea, or see their own reflection in the water.

NICE supports real-time distributed collaboration. M ultiple children can interact with the garden and each other from remote sites. Each remote user's presence in the virtual space is established using an avatar, a graphical representation of the person's body in the virtual world. (See Figure 2.) T he avatars have a separate head, body, and hand that correspond to the user's actual tracked head and hand motions. This allows the environment to record and transmit sufficiently detailed gestures between the participants, such as the nodding of their heads, the waving of their hand, and the exchange of objects. Additionally, voice communication is enabled by a real-time audio connection.

N ICE represents an explicit attempt to blend several learning and pedagogical themes within a single application. The themes-constructionism, exploratory learning, collaboration, and the primacy of narrative- reflect several of the most important educational reform themes of the past three decades.

\subsection{Constructionism and Exploration}

The design of NICE supports the constructivist view that learners assimilate knowledge by engaging in self-directed learning activities that are accomplished through constructive tasks (D ewey, 1966; Papert, 1980). The approach to constructionism taken by N ICE echoes Papert's ideas in two ways. First, the learners can craft the environment within the virtual world. The activities of planting and tending of the garden entail making, manipulating, and exploring objects, systems, and ideas. The plants are simple agents with common rules of behavior based on simplified ecological models. They contain a common set of characteristics that contribute to their growth (such as their age, the amount of water and sunlight they need, and their proximity to other plants). The combination of these attributes determines the health of each plant and its size. The children gradually discover these relationships aided by the direct feedback provided. 
Second, the learners can construct something meaningful to them, such as the narrative.

\subsection{Narrative}

Papert believes that learning takes place when engaged in the construction of a personally meaningful artifact, such as a piece of art, a story, or an interactive computerized object (Papert, 1980). The constructive artifact in NICE is in many ways the garden itself, as well as the stories formed by the kids that participate. O ur original intentions for the narrative development in N ICE stemmed from an earlier project, the Graphical Storywriter (Steiner \& M oher, 1992), a shared workspace where young children can develop and create structurally complete stories.

The stories developed in NICE differ in that they do not achieve closure; rather, they continue to evolve along with the garden. Every action in the environment adds to the story that is being continuously formed. The narrative revolves around tending the garden and the reactions or decisions taken while interacting with the other characters. These interactions are captured by the system in the form of simple sentences such as: "Amy pulls a cloud over the carrot patch and waters it. The tomatoes complain that they are not getting enough water." This story sequence goes through a simple parser, which replaces some of the words with their iconic representations and publishes it on a Web page. (See Figure 3). This gives the story a picturebook look that the child can print to take home. As a tangible product of the virtual experience, this visual output is intended as a way to strengthen the interest and motivation of a student and not so much to challenge reading and writing skills. It is, however, possible that, with further development, a predominantly visual medium such as immersive VR can provide a valuable environment for literary experiences.

\subsection{Collaboration}

O ne of the most important purposes of an educational environment is to promote the social interaction among children located in the same physical space.

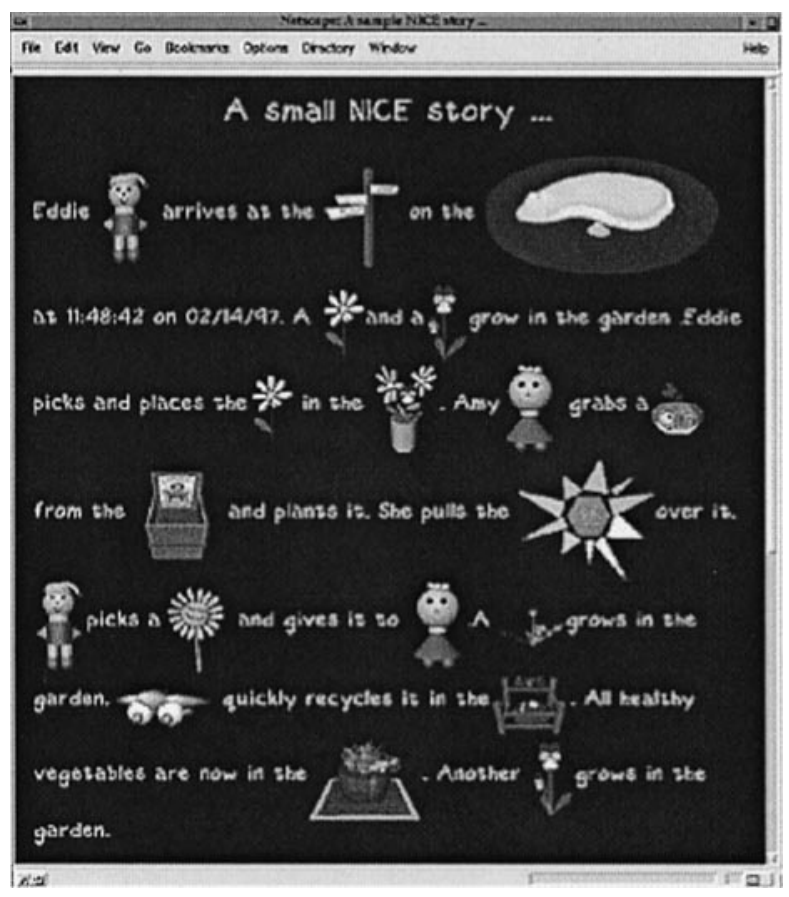

Figure 3. A N ICE story on the web.

Theories that emphasize the importance of social interaction in cognitive growth (Vygotskii, 1978) suggest that successful collaborative learning involves more than the final creation of a learning product. Learning that is contextualized in a social setting may involve verbal interaction, collective decision making, conflict resolutions, peer teaching, and other group learning situations that are characteristic of a classroom setting. With the use of VR technology that supports multiple users in the same physical space, as well as appropriate interaction techniques, a number of kids can participate in the learning activity at the same time, without having to take turns or wear heavy and intrusive hardware devices.

In NICE, the construction of the environment may foster collaboration. The power of the user to modify the environment is manifested on multiple levels, covering the spectrum of available interface options, from bodily to visual to textual representation. Through the use of avatars, geographically separated learners are simultaneously present in the virtual environment. The ability to connect with learners at distant locationsenhanced by visual, gestural, and verbal interaction-can be important to the development of unique collabora- 
tive experiences for both the students and the educators. Teachers or parents can participate, either as members of the groups, or disguised as characters in the environment. This allows teachers to mentor the children in person or to guide parts of the activity from "behind the scenes," acting as simulated virtual characters. They can also determine the pace at which the world evolves; they may choose to see the plants grow very quickly, or, in the case of a school project, extend their growth over the period of a semester.

We explored this notion of a teacher-avatar in the studies we performed with students, as mentioned later.

\subsection{Implementation}

The growth of the plants is handled by a central garden server that can be run on any centrally located computer chosen to maintain the virtual world. The N ICE garden is persistent, as the garden server is constantly running and will attempt to reestablish lost connections in the event of failure. This allows users to casually join in the collaboration whenever they wish. The garden continues to evolve even if no users are present: the plants continue to grow, animals may try to eat the vegetables, and weeds slowly take over the garden and crowd out the other plants.

Since the CAVE library can support heterogeneous

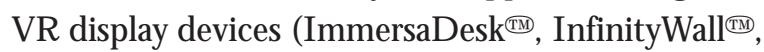
BO O M (iin, fish-tank VR systems) a large number of participants can join in the collaboration from a number of different VR hardware platforms. M ultiple distributed N ICE applications running on separate VR systems are connected via the central garden server which guarantees consistency within the shared virtual environment. In practice, this has been tested successfully with as many as sixteen simultaneous participants on three continents (J ohnson, L eigh, \& Costigan, 1998b).

The networking architecture for the N ICE application was based on previous experience with CALVIN, a networked immersive collaborative environment for designing architectural spaces (Leigh \& J ohnson, 1996). The networking protocols selected were tailored towards the characteristics of VR data and the ability to enter and leave the environment easily from anywhere on the Internet. The networking component also allows clients other than VR interfaces to participate. For example, a recorder client can be connected to the network that records all of the movements and interactions in the virtual environment, allowing the session to be replayed later during evaluation studies. M onitoring clients can be connected to monitor the state of the NICE island and the state of the network. Web-based clients can also connect and cooperate with the VR clients, as explained in the next section.

\section{Extending the Virtual Environment}

Interactivity in N ICE is augmented by providing possibilities to interact with the virtual world without being inside it. The children can check on the progress of the garden from a desktop computer with a Web browser and an Internet connection, seeing who is currently working in the garden and how the various plants are growing. (See Figure 4). They can converse with the other virtual and remote participants by typing in the provided text window, a feature that resembles textbased virtual environments. This feature is currently being enhanced with audio so the desktop users can talk to the immersive VR users.

Children without access to the VR system but with a personal computer and access to the Web may even create their own objects and characters to populate the virtual world. These models are downloaded by the N ICE system automatically and in real time when the users join the collaboration. We are currently working on a VRM L interface to the garden itself. (See Figure 5).

U sing a J ava applet written by R obert Stevenson, students interacting with a two-dimensional version of N ICE on the I nternet can simultaneously share and manipulate the same virtual space as the children in the CAVE. The users of the 2D environment use a traditional mouse-and-icon interface to interact with the garden, but have the same ability to pick and plant as the VR users do. These desktop users see the virtual reality users as $2 D$ icons on their screen, while the VR users see 


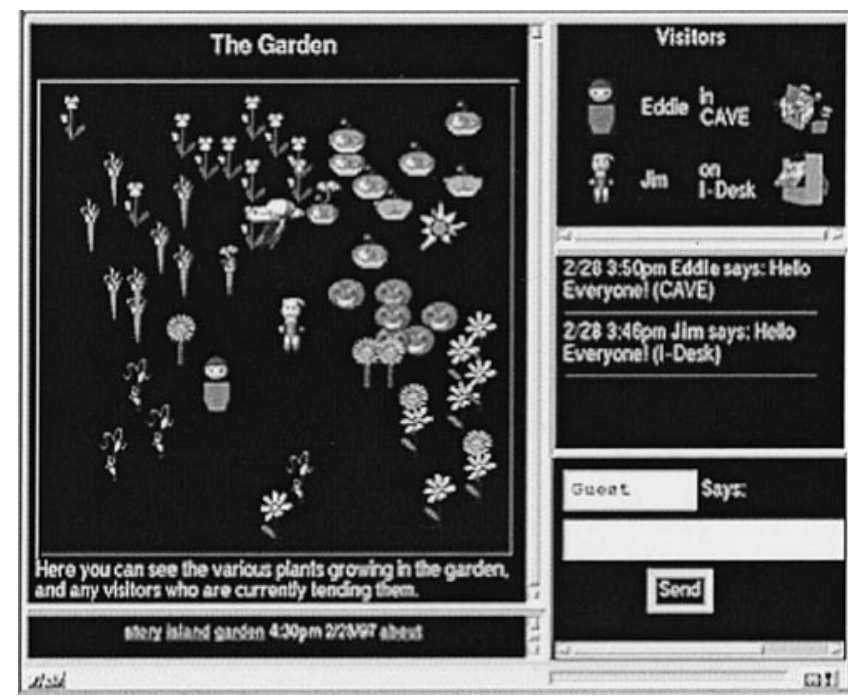

Figure 4. A view of the NICE garden and chat window on the Web.

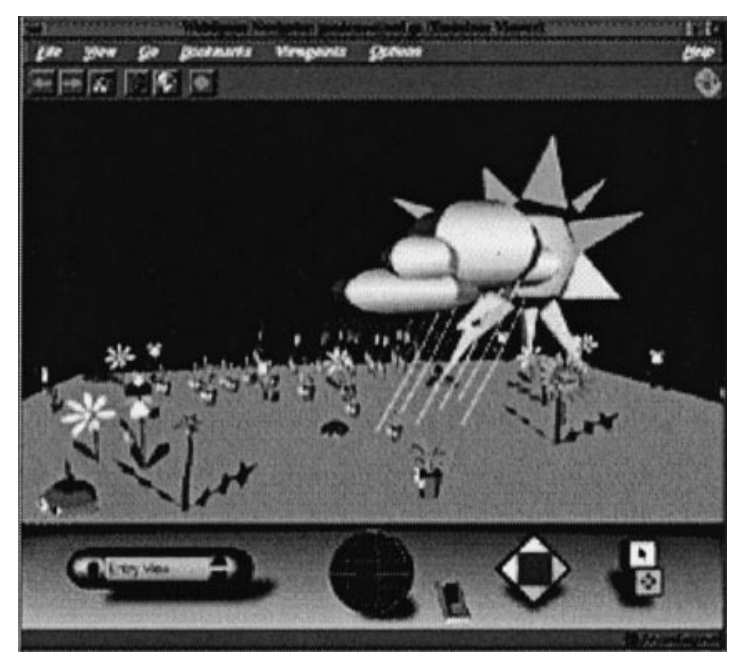

Figure 5. An interface to the NICE garden using a VRM L browser.

the desktop users as 3D avatars in the space. (See Figure $6)$.

Finally, a current prototype is a 2-D interface in which the child, by clicking and dragging icons, can manipulate the ecological model and observe an immediate effect on the growth of the plants and vegetables in the 3-D $V R$ environment. We envision these $2-D$ interfaces as a kind of visual language, allowing the VR worlds to be

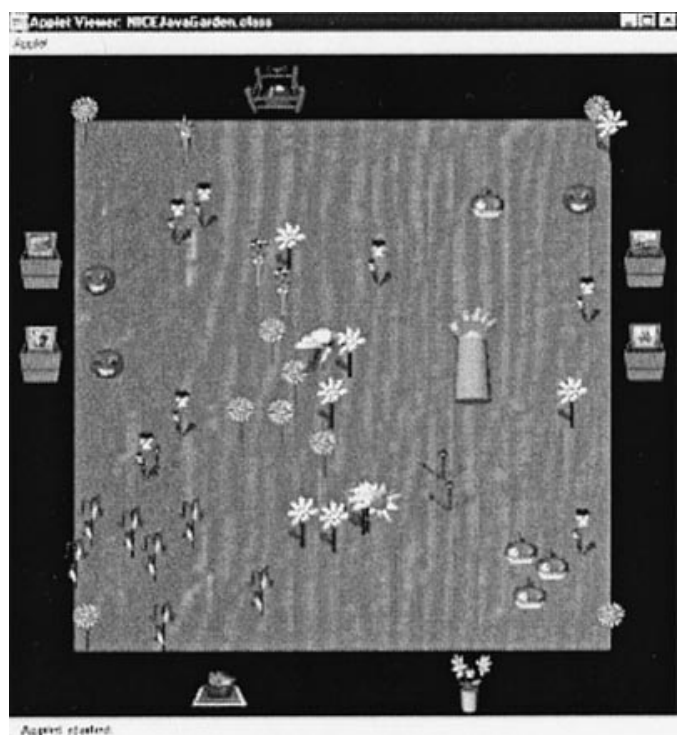

Figure 6. A two-dimensional interface to the NICE garden using a Java applet.

easily programmed by children. V isual languages have shown to be ideal programming languages for young learners because they can map abstract concepts to pictorial elements that they are more familiar with (Soloway, Jackson, Klein, Q uintana, Reed, Spitulnik, Stratford, Studer, Eng, \& Scala, 1996) and can belearned quickly.

Allowing Web-based and desktop participants access to the virtual environment provides several advantages for both the users as well as the researchers and educators. VR hardware is presently expensive and inaccessible to the public. Even when the technology becomes cheaper and more accessible, the time that a child can spend in an immersive virtual environment will still be limited. The Web-based component allows children to sustain their interaction with the virtual world beyond the limited time they can spend in the virtual environment itself. It also allows educators and researchers to participate and evaluate the experience easier. Additionally, this approach holds promise for social interaction by students who are either geographically isolated or who have special needs. 


\section{Evaluation}

It is important to investigate the educational efficacy of VR in specific learning situations and broader learning domains, and to develop new rubrics of educational efficacy that compare it to other approaches. In practice, however, the assessment of VR technology has been focused primarily on its usefulness for training rather than its efficacy for supporting learning in domains with a high conceptual and social content ( $D$ ede et al., 1996; Whitelock, Brna, \& H olland, 1996).

The education world would argue that using paper and pencil, in the form of standardized tests, is not an effective way to evaluate a virtual learning experience. As $V R$ is a dynamic learning tool, evaluation should be tightly coupled with the actual learning process. Following the authentic assessment model, learning in constructivist environments is directly related to its evaluation (Reeves \& O key, 1996). M oreover, considering the immaturity of the field at this time, it is important to apply multiple measures of learning and performance (Rose, 1995).

V irtual reality itself has great potential as a tool for assessment. N etworked VR systems can embed methods for facilitating learner's discourse while in the environment. M entors, disguised as virtual characters, serve as guides and evaluators to answer questions, direct action, ask for clarification, or prompt for interpretation. In addition to recording data such as video and audio while in the virtual environment, it is also straightforward to have one of the networked clients act as a recorder, allowing the entire VR session to be played back in 3-D for further reflection and interpretation (J ohnson et al., 1998). This form of assessment, embedded in the learning process, can provide meaningful reflections on learners' skills and knowledge.

\subsection{Conceptual Framework}

Of particular interest to us was the exploration of the effects of the N ICE virtual environment as well as the overall educational efficacy of VR learning experiences. As a first step, we developed an evaluation framework meant to serve as a prototype for a general evalua- tion framework. The exploratory nature of this study required a sound conceptual framework that would encompass, rather than restrict, the multiple dimensions of the issues that need to be examined in a virtual learning environment. Taking into account the multidimensionality of learning as well as VR as a field, a number of technical, orientational, affective, cognitive, pedagogical, and other aspects were included (L ewin, 1995).

The technical aspect examines usability issues, with respect to interface, physical problems, and system hardware and software.

The orientation aspect examines the relationship of the user to the virtual environment, including navigation, spatial orientation, presence and immersion, and feedback issues.

The affective parameter looks at the user's engagement, likes and dislikes, and confidence in the virtual environment.

The cognitive aspect identifies any improvement of the subject's internal concepts through this learning experience. We tried to evaluate the cognitive parameter in part from within the environment, with the given learning task built into the experience. In N ICE, for example, the teacher-avatar can give goals to the users or ask them questions (e.g., plant and harvest a row of tomatoes). The responses to these activities may reveal what the user understands about the environment while inside it.

Finally, the pedagogical aspect includes the teaching approach: how to gain knowledge effectively about the environment and the concepts that are being taught (in this case, ecology or earth science). With respect to NICE, this aspect is examined in the context of collaboration between students or between teacher-avatars and students. The evaluation framework is summarized in Table 1.

\subsection{Methodology}

The main study sessions were conducted with a total of 52 children: 44 second-grade children from an urban elementary school with an ethnically mixed student population; another 8 children from other schools participated in case studies after the classroom studies were completed. The gender distribution was equal: 26 
Table I. Summary of Evaluation Framework

\begin{tabular}{|c|c|c|}
\hline $\begin{array}{l}\text { Framework } \\
\text { Category }\end{array}$ & I ssue & M easurement \\
\hline Technical & U sability & $\begin{array}{l}\text { Time to learn an interface, comprehension of } \\
\text { instructions, physical and emotional comfort }\end{array}$ \\
\hline O rientation & $\begin{array}{l}\mathrm{N} \text { avigation, spatial orientation, presence and } \\
\text { immersion, and feedback }\end{array}$ & $\begin{array}{l}\text { Time to become immersed and comfortable in } \\
\text { the environment }\end{array}$ \\
\hline Affective & Engagement, preference, and confidence & $\begin{array}{l}\text { L ength of engagement, time to reach fatigue, } \\
\text { reported and perceived enjoyment }\end{array}$ \\
\hline Cognitive & Conceptual change, new skill & $\begin{array}{l}\text { Performance within and outside the environment, } \\
\text { think-aloud and stimulated recall techniques, } \\
\text { oral and written surveys, video documentation }\end{array}$ \\
\hline Pedagogical & $\begin{array}{l}\text { Content general and specific teaching tech- } \\
\text { niques }\end{array}$ & $\begin{array}{l}\text { Collaboration (e.g., turn-taking, conflict, inter- } \\
\text { action), avatar acceptance, comparison of } \\
\text { techniques }\end{array}$ \\
\hline $\begin{array}{l}\text { Collaborative } \\
\text { VR }\end{array}$ & $\begin{array}{l}\text { The added value of collaborative VR to instruc- } \\
\text { tion and learning }\end{array}$ & $\begin{array}{l}\text { Comparisons of instruction and learning within } \\
\text { and outside of collaborative VR environments }\end{array}$ \\
\hline
\end{tabular}

boys and 26 girls. The activities at each evaluation session of N ICE took approximately one to three hours to complete, depending upon whether the tests were conducted with groups or pairs of children. This included time to introduce the activity and organize the students, time to plan the activity beforehand, perform the activity inside the VR environment, and have some time for post-activity questions and discussion. The $V R$ setting in all studies included the CAVE and one or two I mmersaD esks, all linked by an audio connection. The teachers were asked to evaluate the students in their class according to their reading and writing skills, leadership skills, and shyness. The children were then assigned to groups. We tried to keep the groups as equally distributed as possible by selectively matching and assigning the children with strong leadership skills or strong reading and writing skills to different groups. Each class of 22 students was divided into three teams of 7 to 8 students each.

Before beginning the VR experience, the children were asked to complete question sheets. These initial questions attempted to identify each child's relationship to technology, familiarity with gardening, and understanding of simple ecological concepts. We wanted to establish what knowledge and understanding of the con- cepts displayed in the environment the children brought with them to the study.

After completing the questionnaires, each group of students was asked to generate ideas for planning their garden. A large piece of paper containing an overhead view of the garden was given to each group. Four rows of differently colored stickers, each one representing one of the four available vegetables, were provided. The children in each group had to plan where they would plant their vegetables by placing the stickers on the soil area of the garden. A total of forty vegetables were allowed (ten stickers for each kind). After the planning stage, the first team continued onto the CAVE and I mmersaD esk part, while the other teams remained in the room to continue their concept maps. Each team was split into two groups, one for the CAVE and the other for the I mmersaD esk. The two groups collaborated remotely, represented by the avatar of the leader of each group. The leader was assigned randomly by the researchers to avoid conflicts during the VR experience. The leaders were instructed in the use of the wand and were allowed a ten-minute period to practice navigation. Each session lasted an average of thirty minutes. I $n$ addition to the two avatars sharing the same virtual space, an adult acting as teacher was disguised as a girl avatar and was guid- 
ing the groups from another I mmersaD esk. This teacher-avatar was also responsible for keeping the time, keeping the children focused on their planting task, helping them accomplish the garden planned on paper, and encouraging the two groups to think aloud. An audio connection between the three VR sites was established through the use of hidden ambient microphones. O ut of a total of eight groups for each classroom, four groups were of single gender (two all-girls teams, two all-boys teams), and the remaining two were of mixed gender.

Following the virtual experience, an open-ended set of interviews was conducted with the children during which they answered an additional set of questions that related to their impressions and understanding of the environmental relationships in the N ICE garden. The questions included space for open-ended responses and discussion with the researcher, regarding what the children did while in the environment, what they liked or disliked, and what they thought they learned.

After the interview, the groups returned to the room they started out from. Large pieces of white paper were placed on the tables, upon which the students could draw. They were asked to draw the gardens they just created in N ICE. Similar activities also continued in their classrooms after the experiments. The teachers assigned homework reports to describe the VR experience and propose their own virtual worlds. Some of the children from the case studies returned a few more times to participate in NICE at a later time.

\subsection{Observations}

The observed results from the case and classroom studies have been grouped based on the theoretical framework defined previously. These observations have been collected by converging the multiple pieces of data gathered through observation, interviews and questionnaires, and are presented below.

5.3.1 Technical issues. The children exhibited diversity in their use of the interaction device (the wand). The instructions given depended on the situation and the environmental or personal distractions, so they were not exactly the same for everyone. Generally, these instructions started with showing the representation of the virtual hand to the leader, then the use of the joystick for navigation, and finally, once the child was able to move comfortably, the function of the buttons. Learning the functions of the wand lasted from two minutes (for the children who learned quickly) to seven minutes.

After learning how to use the wand, the children's effort was focused on orientation, as noted in the following section. Limitations of the physical design of the wand caused discomfort to young users, as both hands were needed to reach the buttons and press the joystick at the same time. It was expected that the boys would generally be better at using the wand, partly because of their familiarity with similar input devices from playing video and computer games. (According to both parents' and kids' reports, $92 \%$ of the boys play electronic games weekly, as opposed to $42 \%$ of the girls.) The majority of these games have joystick-based interface devices. We did not notice, however, any gender differences in learning to use the wand.

A larger problem was the size of the stereo glasses. D espite the glass-ties used to tighten the glasses on the children's heads, the glasses would still fall off. M ost children had to hold the glasses with their free hand and, when tired of holding them, would just take them off. $\mathrm{N}$ ot only did this contribute to the subjects' fatigue, but also to their level of motivation and excitement. Since the stereo glasses and the wand are an integral part of the virtual experience, these limitations are a current hindrance not only to usability but also to learning.

The children's susceptibility to simulator sickness was not as large as expected. Less than $5 \%$ of the subjects complained about getting a headache or being dizzy during or after the experience, and for most it was so slight that they had not noticed until asked.

Evaluation of the system with respect to its robustness and cost effectiveness for broader use must be put off until the system is in a public locale. The N ICE software is flexible enough to eventually expand into a user-authoring system. To be effective, however, it needs to be used by a small number of learners for an extended period of time. 
5.3.2 Orientation. After learning how to use the wand, the children focused on trying to navigate and orient themselves in the virtual environment. With respect to the classroom groups, this proved to be the effort of the leader and not of the other children in the group, although their mission was to help the leader. The drivers were the only ones focused on the orientation task at hand, as they were the ones navigating, while their movement and the 3-D graphics distracted the other children. The girls seemed slightly better at orienting themselves in the environment, possibly because they were generally more focused and reserved compared to the boys. Even with the case studies, although not nearly to the same extent, there were times when the other child (the one not using the wand) would wander around instead of observing or directing the driver's actions. While it was not expected that all children's full attention would be given at orientation, the result in these studies was that each child came up with their own version of the right direction, voiced them at the same time as the other children and confused the leader, who then individually decided which was the right path to take. As a result, apart from the difficulty in using the joystick for navigation, the leaders exhibited noticeable individual differences in their abilities to interact with the 3-D environment. These differences seemed to relate to their level of independence: the ones pursuing their own goals did well, while the ones who attempted to listen to the others in their group ended up confused and disoriented.

A test for spatial orientation was the ability to find areas in the space, such as a hole that leads to the area under the garden. This was a relatively difficult task, although there were spatial clues: the passage was located near the only set of trees behind one of the garden fences. These were some of the instances in which verbal interaction between the children and teacher seemed to work well, largely because the goal was very specific and required the kids' complete attention.

Another test for orientation was the concept map (the plan of the garden on paper). In the planning stage, students developed different strategies for planting. We wanted to see how they were able to implement this plan in VR. The case studies were more focused; therefore, the children attempted to stick to their plan. With the exception of one boy in the initial study, the children were not successful at completing the task. M ost children began planting as planned, but then changed their plans when running into difficulty. A younger girl who tried following the plan, commented that it was very hard to be precise in separating the vegetables. The teacher-avatar helped her with directions, but that "wasn't enough." The classrooms, on the other hand, hardly even tried to implement the plan, although constantly reminded by the teacher-avatar. Their entire experience was consumed by dealing with the group's behavior. N one of the children admitted that they did not try; rather, they stated that implementing the plan was a difficult task. O ne boy, after seeing the look of the group's final version of the garden asked his group: "how come we didn't get it right?" to receive the overwhelming response "because it was very hard!"

As perceived through observation, most kids felt immersed as indicated by their motion and excitement. Almost all children attempted to "touch" the virtual objects by moving and clasping their hands in the air. This was particularly noticeable in the case of the virtual beam that extended from the user's hand to help point to and select objects. As the beam was always attached to the hand and close to the user, it felt very 3-D to the children. $M$ any leaders waved at the other avatars with the hand that was holding the wand, indicating that they understood the relationship between the wand, their real hand, and the virtual hand.

5.3.3 Affective. M easuring motivation is difficult, as it is indirect. M oreover, in the case of virtual reality, motivation is highly driven by other factors, such as the novelty effect, media hyperboly, and social issues. It is significant to look through these factors and try to identify whether the content taught within this medium is motivating for children, what it is that motivates them, and most importantly, for how long. This was difficult, as all the children were excited before starting, just by the fact that they would experience virtual reality. Therefore, we had to look at their level of extended engagement during the actual experience.

The amount of time the children spent in VR ranged 
from thirty minutes to one and a half hours. Each classroom group, due to time constraints, remained in the experience for about thirty minutes. The case-study subjects, on the other hand, were allowed to stay until they displayed noticeable fatigue, at which point they were asked if they wished to continue. M ost cases wished to remain in N ICE for at least 45 minutes and started getting tired after one to one and a half hours.

Interactive activities ranked high amongst the preferences of the children, as shown by their responses in the post-study questions. Planting was a favorite. An equal number of responses were in favor of the area under the garden. The fantasy was another fundamental driving force for many of the children. M any liked the water (or "swimming"), the rain, sun, umbrellas and sunglasses, and the vegetables. The three things that were most disliked by the children included "the stuff that we had to move with," the "glasses falling off," and the fact that some did not get to drive. M ost (73\%) of the children answered "nothing" to the question "what did you dislike the most?"

The most important issue related to motivation is control. As mentioned in the discussion of orientation, the children that were leading were more on-task and engaged, while all others were distracted and unfocused. This was also perceived, to a lesser degree, with the pairs of children in the case studies: the driver was focused on the task even if that meant only navigation, and was consequently more engaged, while the second child seemed less engaged. The post-experience questions verify these observations: children who were leaders listed that what they enjoyed the most was being the leader, while most others who did not get that chance were very disappointed. M any of these observations are consistent with findings by other researchers in computer-based literature (M alone \& Lepper, 1987).

5.3.4 Cognitive. Examining the cognitive value of a virtual learning environment is very difficult, as there are many other factors that correlate to learning, such as the ones described above. Particularly, distraction, fatigue, and cognitive overhead in mastering the interface influence the outcome. The classroom studies provide good examples of a situation in which all the above took place, and where one cannot derive any conclusions about conceptual learning. The results from the case studies are more promising, as the studies were more focused, prolonged, and with less noise and disorder.

H owever, even in the case studies, little can be concluded as far as learning is concerned. Confidence in using the interface does not necessarily signify understanding of the subject matter. O ne of the boys, for example, who reported playing many hours of video games per week, learned the interface very quickly and easily and had very good navigation and picking skills. H e was interviewed after interacting with VR for about forty minutes. D uring the interview and his post-study questions, it was revealed that he had not perceived the effects of the sun and the rain on the plants, or the function of the umbrellas and sunglasses. This was consistent with his pre-study test, which showed little knowledge of gardening concepts.

To simplify the understanding of the children's knowledge before and after the virtual experience, their responses were grouped into categories. For the pre-study test, three categories were devised according to the children's understanding of simple ecological relationships. The first category included the responses that displayed a very good understanding of gardening concepts: the plants need water and sunlight (i.e., good temperature) and good soil to grow, they wilt or look brown when they are sick, they wilt if they get too much water and dry out when they get too much sun, and the weeds need to be pulled out. Approximately $12 \%$ of the subjects answered in this way. They were also the ones ranked high in reading/ writing skills by the teachers. The second category ( $42 \%$ of the children's answers) included most of the above answers except for a few misconceptions (e.g., water is good but sunlight is bad for plants). The third category included $44 \%$ of the responses, where more than one question included a "don't know" response or a wrong answer (such as "the plants grow down" when they get sunlight, or that weeds need to be planted and watered). Finally, one child could not answer most of the questions.

The answers to the post-study questions were grouped into categories based on the children's under- 
standing of the N ICE model: the plants display umbrellas when they receive too much water and sunglasses when there's too much sun, and the weeds are recycled in the compost heap. The responses here were more difficult to categorize, as many children had trouble synthesizing their learning during post-testing, due to fatigue or excitement, while others misunderstood the questions and answered in the same way as in the pretest, not understanding that the post-questions pertained to the NICE garden in particular.

Approximately seventeen children (35\%) understood, for the most part, the N ICE model. Of these seventeen, thirteen were drivers, and all had done well in their prestudy questions. This shows that most of the leaderschildren that were actively engaged in the task-understood the model of the NICE garden, whereas only a few of the other children perceived it. Approximately $45 \%$ of the children simply answered "they grew" to the questions "what happened when you put the rain over the plants" and "what happened when you put the sun over the plants." Five children answered that they did not know or see what happened while six were tired and did not answer at all.

5.3.5 Pedagogical. The children acted naturally while in NICE, just as they would have at a playground. They played, argued, listened, spoke loudly, and even rested. Very few were curious about the technology, excepting a girl who asked if the screens were made of paper. The children throughout the sessions did not generally perceive the presence of "the computer." As one child put it, "I thought we were going to play with a computer, but this was different." This indicates that perhaps VR may come closer to a "natural" medium for teaching, once technical and technology-specific problems are resolved.

Although children in these studies participated in the VR session longer than in any other educational VR study, it appears that this was not an important factor in the facilitation of learning. We do agree, however, with D ede et al. (1996) who report that spreading lessons over multiple VR sessions appears to be more effective than covering many topics in a single session, as we attempted to do in our studies. R eviews and post-tests from their studies demonstrated that students were better able to retain and integrate information over multiple lessons. This is usually the case in school-based learning as well as being the main concept of life-long learning.

With respect to their pedagogical function in the N ICE studies, collaboration and the narrative are explored further in the following sections.

5.3.6 Collaboration. The classroom studies were set up to encourage intragroup collaboration and intergroup competition, to ensure that each group had an incentive to focus on the task of creating a tended garden. H owever, none of these forms of cooperation occurred. After each group was split (one subgroup to go to one VR system and the other group to the other), the children had to be continuously reminded by the teacher-avatar that they were still one group working on a common goal in the same garden. M ost children, however, continued not to perceive this and regarded the other (remote) half of their group as their competitors. There were multiple instances of the two drivers fighting over who would grab the raincloud, and children from one location yelling at the ones in the other location to step out of "their" garden. As far as the classrooms were concerned, competition contributed to the excitement of the children in the group, but kept them off-task and distracted for nearly the entire experience. Some of the groups even displayed a form of intragroup competition between the leader and other members. This related mainly to the control of the wand. $\mathrm{N}$ otable is the case of one girl who caused constant conflict because she was not the one chosen to be in control. The intent during these studies was to have only one child in each group control the wand. $O$ ur rationale for this was efficiency: it is easier and quicker to teach one subject than all, it is more efficient for one to control while others direct the activity, and it avoids fighting over who will do it.

On the other hand, this efficiency gain might not be helpful in terms of advancing all the students' learning. In the case of the other students, it was evident that the control over their learning and their experience was in the hands of the leader of the group. It was hoped that, in this way, the students would be able to pay more attention to the subject matter by leaving the control of 
the learning situation to the leader. For the child controlling, we supposed that this would not be an advantage, as it could lead to less attention to the subject matter and more to the task of controlling. As noted previously, the opposite was observed in these studies: the leader paid more attention to the subject than did the other less active members of the group.

Contrary to the classroom's behavior, the pairs of children in the case studies displayed excellent collaboration and no competition. In most cases, on-task communication was observed and there was general agreement on actions. Based on these observations, issues regarding the selection and number of members in a group of second-graders must be taken into account for a successful collaborative combination.

For both the classroom as well as the case studies, the teacher-avatar seemed to serve a helpful purpose, especially for giving tips and keeping the students on task. In terms of the classroom children, of course, the teacheravatar consumed most of her time attempting to keep order (not unlike a real classroom).

The system's visual output (a printout of the narrative WWW page) was shown to each group during the interview to help the children reflect on their virtual experience. Each group was represented in the story by the avatar of the leader. Some children did not understand this until it was explained to them while showing them the narrative. M ost were fascinated by the pictorial representations of the characters and vegetables and remembered what they were doing by looking at the story. It is believed that the iconic representation was helpful in giving the groups a general overview of their actions and is worthy of further exploration. An unanticipated function of the story was its use as a spelling aid by two children from different groups. When completing their questions, they consulted the story to find the spelling of certain object names.

\section{Conclusions}

In our view, the NICE project had a number of highly positive outcomes. There was ample evidence that the environment provided a strong sense of presence and immersion; one adult visitor to N ICE commented that it was "the closest I've ever come to the feeling of being inside one of the cartoons I used to watch on televisions when I was a kid." NICE appeared to be a highly successful distributed virtual social space, particularly for those "drivers" who had full access to the input affordances. O n the technical front, the N ICE project provided a driving application for the development of the CAVER N Soft distributed virtual environment architecture (Leigh \& Johnson, 1998).

In retrospect, the most serious shortcoming of N ICE is the inadequacy of its science model. In an attempt to engage children, we introduced elements (umbrellas, sunglasses, and facial expressions) without natural analogs, and misrepresented naturally occurring features (e.g., root systems). These artifacts, deployed in a setting decontextualized from supporting discussion and instruction, may themselves have become the source of misconceptions regarding the underlying growth model we were attempting to teach. The balance among reality, abstraction, and engagement is particularly difficult to achieve; in this case, we likely veered sufficiently from reality to endanger the raison d'être behind the project.

A second source of difficulty, in our opinion, drew from the open-ended exploratory nature of the environment itself. Instead of directing activity toward (and providing affordances for) the discovery of the underlying scientific knowledge, we assumed that the desired learning would take place naturally through exploration and discovery. This lack of direction, both within the environment and in our task charges to users-combined with the novelty of the environment and usability issues associated with the learning of novel control affordances- appeared to obscure the intended learning goals in the eyes of the users.

Finally, collaboration itself proved a double-edged sword. The presence of avatars representing remote users was a strong spur to social interaction, again at the expense of the intended science learning. N ICE supported collaboration through the provision of a shared virtual space, but did little to structure cooperative learning (Slavin, 1980; Johnson \& J ohnson, 1984) in a way that fostered positive interdependence among learners, or supported reflection and planning. Social interac- 
tion became an end unto itself, rather than a mechanism to support learning.

Researchers interested in learning in immersive virtual environments face a difficult challenge. $\mathrm{O} n$ the one hand, there is a strong need for demonstrable "added value" to learning associated with the use of VR technologies; in spite of our optimism regarding the ultimate broad availability of these technologies, there is little reason to bring $V R$ technology to bear on learning goals that are already well met by conventional pedagogy. At the same time, however, it is difficult to conceive, much less conduct, an experiment whose results would be sufficiently generalizable to sway skeptics. Certainly, there can be no experiment that ascribes specific learning value to the technology itself; the failure of an experiment to demonstrate added learning value would be due at least as much to the application as to the underlying technology.

There is a place for controlled experimental studies of learning in immersive virtual environments; we need more objective success stories. But the primary focus of this research domain, particularly in the case of younger children, should be directed toward the development and informal empirical evaluation of novel learning applications. In both cases, we believe that researchers should focus their attention on learning problems that meet four criteria:

1. The learning goal must be important; i.e., it must be identified as a component of adult scientific (or other) "literacy," as reflected in national learning goals, standards, or benchmarks, such as those published by the $\mathrm{N}$ ational $\mathrm{C}$ ouncil of Teachers of $M$ athematics or the American Association for the Advancement of Science.

2. The learning goal must be hard. We probably don't need VR to teach simple addition; in hindsight, we probably don't need it to teach simple facts about plant growth. I nstead, we should focus on deep learning problems: learning that requires the rejection of inadequate and misleading models based on everyday experience, that has proven resistant to conventional pedagogy, and that is the source of persistent adult misconceptions.
3. The learning goal must be plausibly enhanced by the introduction of immersive VR technologies. The most obvious plausible domains are those involving solid models; on its face, VR technology would seem to offer more to learning about molecular models than the capitals of the U .S. states. But three-dimensional representation may not be the most important quality that VR brings to bear; there is reason to believe that the ability of $V R$ to situate its users in an alternative cognitive frame of reference may be its most valuable contribution to learning.

4. Finally, VR-based learning environments must be informed by contemporary research in the learning sciences, by contemporary practice in education, and by the practical realities of school organization and funding. Research conducted outside these contexts runs the risk of irrelevancy.

\section{Acknowledgements}

We wish to thank all the teachers and the students and their parents for participating in the user studies, the members of the original "yet another world" group for their valuable discussions, and all of the members of the E lectronic V isualization $L$ aboratory and I nteractive Computing Environments $L$ aboratory for their patience and support. We would especially like to thank Jim Costigan for his help.

The $V R$ research, collaborations, and outreach programs at $E V L$ are made possible through major funding from the $\mathrm{N}$ ational Science Foundation, the D efense Advanced R esearch Projects Agency, and the $U$.S. D epartment of Energy (specifically N SF awards CDA-9303433, CDA-9512272, N CR-9712283, CDA-9720351, and the N SF ASC Partnerships for Advanced Computational I nfrastructure program). The CAVE and ImmersaD esk are trademarks of the Board of Trustees of the $U$ niversity of Illinois.

The continuation of this research is funded by a N SF L earning and Intelligent Systems grant, investigating how VR can be used to help teach concepts that are counterintuitive given the learner's current mental models. 


\section{References}

Allison, D ., Wills, B., Bowman, D ., Wineman, J., \& H odges, L. (1997). The virtual reality go rilla exhibit. IEEE Computer Graphicsand A pplications, 30-38.

AAAS: A merican Association for the Advancement of Science (1992). Science for all Americans: A project 2061 report on literary goals in science, mathematics, and technology. (Technical Report). AAAS, Washington, D.C.: AAAS Publication.

Bricken, M . (1991). Virtual reality learning environments: Potentials and challenges. Computer Graphics 25(3), 178-184.

Bricken, M ., \& Byrne, C . (1993). Summer students in VR: A pilot study. Virtual R eality: A pplications and Explorations. A cademic Publishers Professional, 178-184.

Brooks, F. (1998). Virtual reality in education: Promise and reality panel statement. In ProceedingsI EEE Virtual R eality A nnual I nternational Symposi um (VR A I S '98), 208.

Clark, R. E. (1983). Reconsidering research on learning from media. R eview of Educational R esearch, 53, 445-460.

Cromby, J., Standen, P., \& Brown, D . (1995). U sing virtual environments in special education. VR in the Schools 1(3), 1-4.

Cruz-N eira, C ., Sandin, D. J ., \& D eFanti, T. A. (1993). Surround-screen projection-based virtual reality: The design and implementation of the CAVE. In Proceedings of A CM SI GGRAPH '93, 135-142.

Cuban, L. (1986). Teachers and M achines: The Classroom U se of Technology Since 1920. N ew York: Teachers C ollege Press.

D ede, C. (1998). Virtual reality in education: Promise and reality panel statement. In ProceedingsI EEE Virtual R eality A nnual I nternational Symposium (VR A IS'98), 208.

D ede, C., Salzman, M ., \& L oftin, B. (1996). ScienceSpace: $V$ irtual realities for learning complex and abstract scientific concepts. In Proceedings I EEE Virtual R eality A nnual I nternational Symposium (VR A IS '96), 246-253.

D ewey, J. (1966). D emocracy and Education. N ew York: Free Press.

Gay, E ., \& Greschler, D. (1994). Is virtual reality a good teaching tool? Boston Computer M useum.

Johnson, D. W., \& Johnson, R. T. (1984). Cooperative Learning. $\mathrm{N}$ ew Brighton, $\mathrm{M} \mathrm{N}$ : Interaction Book $\mathrm{Co}$.

Johnson, A., Roussos, M ., L eigh, J., Vasilakis, C., Barnes, C., \& M oher, T. (1998). The N ICE project: Learning together in a virtual world. In Proceedings of VR AIS '98, 176-183. Johnson, A., Leigh, J., \& Costigan, C. (1998b). M ultiway tele-immersion at supercomputing '97. I EEE Computer Graphicsand A pplications, 18(4).

L eigh, J., \& Johnson, A. (1996). Supporting transcontinental collaborative work in persistent virtual environments. IEEE Computer Graphicsand A pplications, 16(4), 47-51.

L eigh, J., J ohnson, A., \& D eF anti, T. (1998). Issues in the design of a flexible distributed architecture for supporting persistence and interoperability in collaborative virtual environments. In Procedings of Super computing '97, 1997.

Lewin, C. (1995). Test driving CARS: Addressing the issues in the evaluation of computer assisted reading software. Proceedings of I nternational C onference on Computersin Education, 452-459.

M alone, T. W., \& Lepper, M . R. (1987). M aking learning fun: A taxonomy of intrinsic motivations for learning. In A ptitude, Learning, and Instruction: Cognitiveand A ffective Process A nalyses. H illsdale, N J : L awrence Erlbaum Associates.

N CTM : N ational C ouncil of Teachers of M athematics (1989). Curriculum and Evaluation Standards for School M athematics.

Papert, S. (1980). M indstorms. C hildren, Computers, and Powerful I deas. N ew York: Basic Books, I nc.

Provenzo, E. F. (1991). Video K ids. Making Sense of N intendo. Cambridge, M A: H arvard U niversity Press.

Reeves, T. C., \& O key, J. R. (1996). Alternative assessment for constructivist learning environments. Constructivist Learning Environments: CaseStudiesin I nstructional D esign. E ducational Technology Publications.

Rose, H . (1995). A ssessing learning in VR : Towards developing a paradigm virtual reality roving vehicles(VRRV) project. Technical Report TR-95-1, H uman Interface Technology Laboratory- $U$ niversity of Washington.

Roussos, M. (1997). I ssuesin the design and evaluation of a virtual realitylearning envir onment. (M aster's thesis, U niversity of Illinois at C hicago).

Roussos, M., Johnson, A., Leigh, J., Barnes, C., Vasilakis, C., \& M oher, T. (1997). The N ICE project: N arrative, immersive, constructionist/ collaborative environments for learning in virtual reality. Proceedings of ED-M ED IA / ED-TELEC OM '97, 917-922.

Roussos, M., Johnson, A., Leigh, J., Vasilakis, C., Barnes, C., \& M oher, T. (1997b). NICE: Combining constructionism, narrative, and collaboration in a virtual learning environment. C omputer Graphics, 31 (3), 62-63.

Slater, M ., \& Wilbur, S. (1997). A framework for immersive virtual environments (FIVE): Speculations on the role of 
presence in virtual environments. Presence: Teleoperatorsand Virtual Environments6(6), 603-616.

Slavin, R. E. (1980). Cooperative learning. R eview of Educational R esearch, 50(2), 315-342.

Soloway, E., Jackson, S., K lein, J ., Q uintana, C., R eed, J ., Spitulnik, J., Stratford, S., Studer, S., Eng, J., \& Scala, N.

(1996). L earning theory in practice: C ase studies of learnercentered design. In Proceedings of A C M Conference on $\mathrm{H}$ uman Factorsin Computing Systems ( CH I '96) . (pp. 189196). N ew York: ACM Press.

Steiner, K., \& M oher, T. (1992). Graphic StoryWriter: An interactive environment for emergent storytelling. I $n$ Proceedings of A CM CH I '92 C onference on $\mathrm{H}$ uman Factorsin Computing Systems (CH I '92). (pp. 357-364). N ew York: ACM Press.
Vygotskii, L. (1978). M ind in Society: The D evelopment of $\mathrm{H}$ igher Psychol ogi cal Processes C ambridge, M A: H arvard U niversity Press.

Whitelock, D ., Brna, P., \& H olland, S. (1996). What is the value of virtual reality for conceptual learning?: Towards a theoretical framework. In Proceedings of European Conference on A rtificial I ntelligence in Education, 136141.

Winn, W. (1993). A conceptual basisfor educational applications of virtual reality. Technical Report TR-93-9, H uman I nterface Technology L aboratory-U niversity of Washington.

Youngblut, C . (1998). Educational uses of virtual reality technology. Technical Report ID A D ocument D-2128, Institute for D efense Analyses, Alexandria, VA. 